TecnoHumanismo. Revista Científica

Setiembre - Noviembre 2021

Volumen 1 / No.3

ISSN: $2710-2394$

pp. $522-536$

https://doi.org/10.53673/th.v1i12.88

https://tecnohumanismo.online

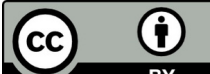

\title{
Comunidades de práctica virtuales en educación superior universitaria
}

\author{
Virtual Communities of Practice in University Higher Education \\ Comunidades virtuais de prática no ensino superior universitário
}

\section{ARTÍCULO GENERAL}

\author{
Paola Elena Vicente Ataurima \\ paola.vicente.ataurima@gmail.com \\ https://orcid.org/0000-0003-1030-8917 \\ Universidad Nacional Mayor de San Marcos, Lima, Perú
}

Recibido 07 de Abril 2021 | Arbitrado y aceptado 19 de Junio 2021 | Publicado en 03 Setiembre 2021

\section{RESUMEN}

Este artículo tuvo por objetivo conocer sobre el rol de las comunidades de práctica virtuales en educación superior universitaria. Para ello, se consideró una investigación de tipo documental, con la revisión de literatura, se hizo consultando las bases de datos como Proquest, Scopus y Science Direct. Con palabras clave como: comunidad de práctica virtual; y educación superior universitaria. Los criterios de inclusión fueron: que sean artículos de revistas indexadas, que la antigüedad de la publicación no sea mayor a 5 años y que la temática guarde relación con la variable comunidad de práctica. Con ello, se recabaron 15 artículos que contaban con lo especificado. Se concluyó con el cumplimiento de este, que fue reflexionar el rol de las comunidades de práctica virtuales en contextos universitarios. Las comunidades de práctica virtuales se han convertido en una herramienta útil para el intercambio de conocimiento y experiencias en los últimos años. Además, aportan ventajas que se añaden a las de las tradicionales. La construcción colectiva de conocimiento que se logra en una $\mathrm{CP}$ tiene un impacto positivo en las prácticas profesionales de los docentes, así como también tiene efectos positivos en el desarrollo académico de los alumnos.

Palabras clave: Comunidad de práctica; Comunidad de práctica virtual; educación superior universitaria; docentes; universitarios.

\section{ABSTRACT}

This article aimed to learn about the role of virtual communities of practice in university higher education. For this, a documentarytype research was considered, with the literature review, it was done by consulting databases such as Proquest, Scopus and Science Direct. With keywords such as: virtual community of practice; and university higher education. The inclusion criteria were: that they be articles from indexed journals, that the age of publication is not more than 5 years and that the subject is related to the community of practice variable. With this, 15 articles were collected that had what was specified. It was concluded with the fulfillment of this, which was to reflect on the role of virtual communities of practice in university contexts. Virtual communities of practice have become a useful tool for the exchange of knowledge and experiences in recent years. In addition, they provide advantages that are added to those of the traditional ones. The collective construction of knowledge that is achieved in a CP has a positive impact on the professional practices of teachers, as well as positive effects on the academic development of students.

Keywords: Community of practice; Virtual community of practice; university higher education; teachers; university students.

\section{RESUMO}

Este artigo teve como objetivo conhecer o papel das comunidades virtuais de prática no ensino superior universitário. Para isso, foi considerada uma pesquisa do tipo documental, com a revisão da literatura, realizada por meio de consulta a bancos de dados como Proquest, Scopus e Science Direct. Com palavras-chave como: comunidade virtual de prática; e ensino superior universitário. Os critérios de inclusão foram: que sejam artigos de periódicos indexados, que a idade de publicação não seja superior a 5 anos e que o assunto esteja relacionado à variável comunidade de prática. Com isso, foram coletados 15 artigos que possuíam o que foi especificado. Concluiu-se com o cumprimento deste, que consistiu em refletir sobre o papel das comunidades virtuais de prática em contextos universitários. Comunidades virtuais de prática tornaramse uma ferramenta útil para a troca de conhecimentos e experiências nos últimos anos. Além disso, apresentam vantagens que se somam às tradicionais. A construção coletiva do conhecimento que se realiza em um CP tem um impacto positivo nas práticas profissionais dos professores, bem como no desenvolvimento acadêmico dos alunos.

Palavras-chave: Comunidade de prática; Comunidade virtual de prática; ensino superior universitário; professores; Estudantes universitários. 


\section{Introducción}

Una de las características de la sociedad contemporánea es el empleo del conocimiento generado en el seno de las universidades en los procesos productivos, considerándose que el factor más relevante ya no es la disponibilidad de capital, mano de obra, materias primas o energía, sino el uso intensivo del conocimiento y la información (García et al., 2008). Por otro lado, las universidades como institución social se han convertido en el instrumento para medir el progreso y el desarrollo, sin embargo, al considerar la realidad actual, debería de incluirse entre sus metas, la preparación de capacidades para vivir en medio de la incertidumbre, transformar y provocar cambios (Paz et al., 2016).

En ese contexto, de acuerdo con Li et al., (2009) la sola interacción entre compañeros en el lugar de trabajo o estudio, fomenta el aprendizaje y el intercambio de información. Surge entonces el término comunidades de práctica $(\mathrm{CoP})$, que constituye una visión contemporánea surgida a finales de la década de 1980, y Lave \& Wenger en 1991, como pioneros en investigaciones comunitarias, formularon la primera aproximación teórica, al observar que la mayor parte del aprendizaje se produce en las relaciones sociales en el lugar de trabajo y no en un aula. De su primer trabajo aun cuando es en el área laboral, concluyen que, las interacciones informales se convierten en el medio para que los profesionales mejoren la práctica y generen nuevas formas de abordar los problemas recurrentes.

El enfoque de Lave \& Wenger, va evolucionando, así en su libro de 1998, dentro del enfoque empresarial, se desplaza hacia el crecimiento personal y la trayectoria de la participación de los individuos dentro de un grupo, y en su publicación del 2002, la CoP es ya una herramienta de gestión para optimizar la competitividad de una organización (Li et al., 2009)

Wenger y Snyder (2000) revisando las características de una CoP, como herramienta de gestión en una organización, la definen considerando tres dimensiones: dominio, comunidad y práctica. El dominio, con la base y el ambiente, refiere a la competencia mínima que diferencia a los miembros de los que no lo son, y traza los límites que permiten a los miembros decidir qué vale la pena compartir y cómo presentar sus ideas. La comunidad, es la estructura social que facilita el aprendizaje a través de las interacciones y las relaciones con los demás. La práctica es el 
conocimiento específico que la comunidad comparte, desarrolla y mantiene, sean las ideas, experiencias, documentos, información y las formas de cómo abordar los problemas. Los autores consideran que, en una $\mathrm{CoP}$ madura, cuando estos tres elementos funcionan bien, se puede optimizar tanto la creatividad como la difusión de los conocimientos.

La conceptualización anterior sustenta que el aprendizaje al interior de la CoP es directamente proporcional a la participación de sus integrantes. Las relaciones en esta forma de asociación permiten profundizar en los niveles de identidad grupal y trascienden una simple trasmisión de contenidos e información. Este rasgo distintivo sustenta la idea de que el proceso de aprendizaje y de pertenencia, constituye la esencia para compartir valores, creencias, lenguajes, entre otros (Wing Lai et al., 2006).

La CoP como una red social persistente y sustentable de individuos que comparten y desarrollan una base de conocimientos superpuesta, un conjunto de creencias, valores, historia y experiencias centradas en una práctica y / o empresa común (Barab et al., 2002), puede aplicarse de forma exitosa, como estrategia para la gestión del conocimiento en diferentes entornos, al demostrar su valor, cuando guía los procesos de innovación, creación o reformulación de prácticas, resolución de problemas críticos, facilitando la transferencia de mejores prácticas, desarrollando habilidades profesionales, reclutando y reteniendo talentos condiciones que pueden dar apoyo al ámbito educativo (Wenger \& Snyder, 2000; Scarso et al., 2009).

De acuerdo con Wenger (2000) los individuos que participan en una CoP deben querer compartir conocimiento, perfeccionar su práctica, construir redes de trabajo eficaces, para lograr dar respuestas a sus necesidades e intereses. Estas interacciones, necesariamente no se complementan con un solo individuo actuando, se necesita de otras personas que se involucren para ofrecer respuestas, uniéndose en diferentes proyectos y construyendo un repertorio compartido (Hara, 2009).

En el ámbito educativo, estos procesos no son muy diferentes, se reconoce que, del diálogo o grupos de discusión, motivan la reflexión y contribuye a que los aprendizajes sean efectivos, aun cuando hay choque de diferentes puntos de vista, y surjan controversias, discrepancias o conflictos. En una institución educativa, las CoP pueden darse en cualquier momento, solo requiere que un grupo de personas de modo informal compartan sus experiencias o reflexiones acerca de un trabajo, y como 
resultado de esta interacción, los estudiantes pueden modificar su comportamiento, sus prácticas o ideas (Castellanos, 2015).

De acuerdo con Castellanos (2015) las personas que conforman una comunidad, aprenden de forma efectiva, cuando la práctica de sus experiencias los une. Así, cuando se logra una práctica compartida, es relevante el proceso de aprendizaje en grupo. De esta forma, puede darse la idea de crearse CoP los cuales amplían el ámbito formativo dentro de las instituciones educativas.

Basado en la noción de las CoP de Wenger, ocurre similar en los miembros ligados a un contexto formativo, cuando los maestros, consejeros, docentes, investigadores, directivos o estudiantes; discuten, identifican y comparten prácticas que responden a realidades académicas y/o sociales y ocurren experiencias dinámicas de aprendizaje. Como grupo de individuos donde todos bajo un conjunto de normas, diseñadas sobre principios de calidad, caracterizan lo que sucede dentro de sus paredes, con el principal objetivo de desarrollar aprendizajes efectivos en los estudiantes (Flórez \& Fernández, 2021). Al considerar el entorno universitario, motivó como objetivo de este estudio, conocer el rol de las comunidades de práctica virtuales en educación superior universitaria.

\section{Materiales y métodos}

Se planteó un estudio de revisión tipo documental, mediante la búsqueda de artículos de investigación publicados en revistas de indexación reconocida, que estuviesen relacionados con CoP en el ámbito educativo universitario, empleando como criterios de búsqueda: artículos en español o inglés; publicados en revistas de acceso abierto, con indexación reconocida como Scopus, Web of Science, Elservier, ProQuest, Scielo, o Latindex; en los últimos cinco años. En respuesta a palabras clave como: comunidad de práctica; comunidad virtual; educación superior universitaria; combinados entre sí utilizando los operadores boléanos "and" y "or".

La búsqueda se realizó en Crossref metada (https://search.crossref.org/), con el filtro temporal establecido y posteriormente se cotejó la indexación de la revista a fin de seleccionar los artículos. 


\section{Resultados}

Se revisaron los trabajos y se tomó nota de los objetivos, las variables y resultados de los estudios, encontrándose 15 artículos que cumplen con los criterios, de inclusión, los cuales se detallan en la Tabla 1.

Tabla 1. Listado de artículos relacionados con comunidad de práctica virtual, publicados entre 2017 y 2020

\begin{tabular}{|c|c|c|}
\hline Autor, año. Objetivo & Variables & Resultados \\
\hline $\begin{array}{l}\text { Bores et al. } 2018 \text { a } \\
\text { Analizar una comunidad de } \\
\text { práctica virtual no formal de } \\
\text { Educación Física (Re)Produce, } \\
\text { como herramienta para la } \\
\text { formación del profesorado }\end{array}$ & $\begin{array}{l}\text { Desarrollo profesional } \\
\text { docente; comunidad } \\
\text { de práctica virtual }\end{array}$ & $\begin{array}{l}\text { Hay ventajas y problemas } \\
\text { en la asincronía y ubicuidad; } \\
\text { la interacción escrita y la } \\
\text { flexibilidad y el carácter } \\
\text { voluntario y abierto de la } \\
\text { comunidad. }\end{array}$ \\
\hline $\begin{array}{l}\text { Bores et al. } 2018 \mathrm{~b} \\
\text { Identificar las motivaciones y } \\
\text { las barreras que señalan los } \\
\text { participantes de (Re)Produce, } \\
\text { una comunidad de práctica } \\
\text { virtual de docentes de } \\
\text { Educación Física. }\end{array}$ & $\begin{array}{l}\text { Barreras y } \\
\text { motivaciones; } \\
\text { comunidad de práctica } \\
\text { virtual de docentes. }\end{array}$ & $\begin{array}{l}\text { Gran diversidad de } \\
\text { motivaciones para la } \\
\text { participación. Las barreras } \\
\text { más frecuentes son la } \\
\text { escasez de tiempo para } \\
\text { dedicar a (Re)Produce, la } \\
\text { complejidad y la densidad } \\
\text { de los textos producidos en } \\
\text { la plataforma y el miedo y el } \\
\text { sentimiento de inferioridad. }\end{array}$ \\
\hline $\begin{array}{l}\text { Bedoya-González et al. } 2018 \\
\text { Este artículo da cuenta de los } \\
\text { procesos de con formación y } \\
\text { dinamización de una CoP de } \\
\text { docentes de L2, interesados en } \\
\text { compartir y construir } \\
\text { conocimiento acerca de los } \\
\text { aspectos didácticos y } \\
\text { tecnológicos de la integración } \\
\text { de las TIC a la enseñanza de } \\
\text { L2 }\end{array}$ & $\begin{array}{l}\text { Comunidad de } \\
\text { práctica, formación de } \\
\text { docentes, lenguas } \\
\text { extranjeras L2 }\end{array}$ & $\begin{array}{l}\text { Hay un impacto positivo en } \\
\text { el interés y la apropiación } \\
\text { de las TIC en los miembros } \\
\text { del núcleo y algunos de los } \\
\text { miembros activos. Un } \\
\text { desafío constante de la } \\
\text { comunidad fue el de integrar } \\
\text { a más miembros del grupo } \\
\text { periférico en las actividades } \\
\text { presenciales y virtuales. }\end{array}$ \\
\hline
\end{tabular}


Arroyo et al. 2018

1. Examinar aspectos teóricos sobre los entornos virtuales de aprendizaje en el contexto universitario e 2 . identificar las características de los entornos virtuales de aprendizaje en el Ecuador

Aldana y Martínez, 2018

El artículo demuestra cómo personal escolar aprovechó las reuniones de proyectos de investigación y controles con asistentes para compartir mejores prácticas y desafíos comunes entre escuelas y roles cuando se trabaja con estudiantes recién llegados
Entornos virtuales de aprendizaje; comunidades de práctica; docentes
Se determinaron las características y beneficios de dichos entornos y se analizaron las ventajas que traería su uso intensivo en la comunidad de práctica de docentes universitarios del Ecuador.

Se subraya la necesidad crítica no solo del desarrollo profesional, sino también de la oportunidad para que maestros, consejeros y administradores resuelvan problemas y colaboren para desarrollar una experiencia educativa académica y social que aproveche los fondos del conocimiento de los recién llegados.

El análisis mostró cómo las publicaciones de investigación anteriores a 2015 han utilizado el marco de la CoP de Wenger para investigar el aprendizaje en línea / combinado en la educación superior y en el desarrollo profesional.

Los estudiantes, al final de sus títulos, dijeron que buscaron ayuda de asesores profesionales, servicios de apoyo para discapacidades, consejeros, asesores. Todos estos eran participantes de la comunidad con los que los estudiantes no tenían afiliación inicial y, por lo tanto, el alumno debía buscarlos de forma independiente. 


\begin{tabular}{|c|c|c|}
\hline $\begin{array}{l}\text { De Carvalho-Folho et al. } 2020 \\
\text { Compartir } 12 \text { consejos para } \\
\text { implementar una comunidad de } \\
\text { práctica para el desarrollo } \\
\text { docente. }\end{array}$ & $\begin{array}{l}\text { comunidad de } \\
\text { práctica; desarrollo } \\
\text { docente }\end{array}$ & $\begin{array}{l}\text { Las CoP son una estrategia } \\
\text { eficaz para reunir a los } \\
\text { miembros de la facultad } \\
\text { comprometidos con las } \\
\text { actividades de enseñanza y } \\
\text { aprendizaje mientras se crea } \\
\text { un entorno seguro y de } \\
\text { confianza. }\end{array}$ \\
\hline $\begin{array}{l}\text { Cruess et al. } 2018 \\
\text { Proporcionar una base teórica } \\
\text { para las múltiples actividades } \\
\text { educativas que tienen lugar } \\
\text { dentro de la comunidad, } \\
\text { ayudando así a crear un } \\
\text { enfoque teórico integrado. }\end{array}$ & $\begin{array}{l}\text { Medicina; comunidad } \\
\text { de práctica; educación } \\
\text { médica }\end{array}$ & $\begin{array}{l}\text { Las iniciativas educativas } \\
\text { que resultan de este enfoque } \\
\text { incluyen agregar } \\
\text { comunidades de práctica a } \\
\text { la base cognitiva; involucrar } \\
\text { activamente a los } \\
\text { estudiantes para que se unan } \\
\text { a la comunidad; creando una } \\
\text { comunidad acogedora. }\end{array}$ \\
\hline $\begin{array}{l}\text { Kensington-Miller, } 2018 \\
10 \text { nuevos académicos de una } \\
\text { variedad de disciplinas } \\
\text { participaron en una comunidad } \\
\text { de práctica, conocida como } \\
\text { Catalyst, para ayudarlos a } \\
\text { encontrar su lugar dentro de la } \\
\text { universidad. }\end{array}$ & $\begin{array}{l}\text { Comunidad de } \\
\text { práctica } \\
\text { multidisciplinaria; } \\
\text { tutoría de pares. }\end{array}$ & $\begin{array}{l}\text { Trabajar en colaboración } \\
\text { fuera de sus disciplinas } \\
\text { brindó espacio para } \\
\text { aprender sobre las } \\
\text { expectativas institucionales } \\
\text { y departamentales, pero lo } \\
\text { que es más importante, la } \\
\text { oportunidad de discutir } \\
\text { temas difíciles que a } \\
\text { menudo surgen para los } \\
\text { nuevos académicos en un } \\
\text { entorno de apoyo. }\end{array}$ \\
\hline $\begin{array}{l}\text { Bosman y Volglewede, } 2019 \\
\text { Describir la organización y los } \\
\text { resultados de las comunidades } \\
\text { de práctica de profesores por } \\
\text { cuatro semestres en los que los } \\
\text { participantes aprendieron sobre } \\
\text { estrategias de instrucción } \\
\text { basadas en la investigación }\end{array}$ & $\begin{array}{l}\text { Comunidad de } \\
\text { práctica docente; } \\
\text { prácticas en el aula }\end{array}$ & $\begin{array}{l}\text { Se descubrió que las } \\
\text { observaciones de los } \\
\text { compañeros inspiraron a los } \\
\text { participantes a adoptar } \\
\text { nuevas estrategias de } \\
\text { instrucción. }\end{array}$ \\
\hline
\end{tabular}


Vegessi y Shaw, 2018

Explorar la contribución de la comunidad de práctica al desarrollo de los estudiantes, el logro de los atributos de los graduados de la Junta de Acreditación de Ingeniería Canadiense (CEAB) y el desarrollo de un ecosistema de innovación.

Hafeez et al. 2019

Examinar cómo los emprendedores se involucran en una comunidad virtual de práctica $(\mathrm{VCoP})$ para compartir conocimientos.
Innovación, liderazgo en ingeniería; comunidad de práctica

Intercambio de conocimientos; emprendedores; comunidad virtual de práctica (VCoP)
Liberatore et al. 2018

Describir el uso de las redes sociales para apoyar a los participantes de la Encuesta de aves de jardín de Nueva Zelanda y examinar las interacciones del grupo en su primer año a través de la lente de una comunidad de práctica
La comunidad de práctica del curso de diseño de procesos ha cambiado con el tiempo y la motivación para la participación en la comunidad ha pasado de la competencia de evaluación comparativa a la competencia de innovación que respalda la innovación de los estudiantes y el desarrollo de la capacidad de liderazgo.

Los emprendedores usan mensajes cortos o medianos para contribuir a las discusiones. Además, los autores encuentran que los miembros de alto nivel y los moderadores de discusiones juegan un papel importante en encender el comportamiento de "reciprocidad" para estimular el interés de la comunidad con la discusión del tema.

El examen de las tres características centrales y los siete principios para el cultivo de una CoP (Wenger et al. 2002) respalda nuestra afirmación de que el grupo de Facebook NZ-GBS está funcionando como una $\mathrm{CoP}$ activa. Los miembros han utilizado al grupo para compartir entusiasmo, ideas y conocimientos sobre las aves de jardín de Nueva Zelanda. 
Costino, 2018

Describe un modelo de comunidad de práctica de desarrollo profesional consciente de la identidad que involucra al profesorado en un enfoque académico de la ciencia del aprendizaje y la enseñanza basada en la evidencia y el desarrollo curricular
Desarrollo del profesorado; modelo interseccional; comunidad de práctica; aprendizaje del profesorado
Necesidad de considerar en la CoP la noción de que se requiere un aprendizaje sólido del profesorado para un cambio institucional con mentalidad de equidad. El modelo descrito aquí contrasta radicalmente con el modelo de taller de "una sola vez" que históricamente ha caracterizado el desarrollo del profesorado en la educación superior.

\section{Discusión}

A lo largo de la revisión se encontraron investigaciones que abordaron la conceptualización de la $\mathrm{CoP}$, en primer lugar, alegando que estas comunidades han crecido en número y calidad desde la década de los años noventa, y pese a la existencia previa de algunas redes de trabajo, su número e influencia se ha incrementado en los últimos año (Aldana y Martínez, 2018; Arroyo et al., 2018; Bores et al., 2018 a); y se han definido, además, tipos de comunidades, roles dentro de las comunidades y las formas más exitosas de crear y gestionar cada una de ellas (Bedoya et al., 2018).

Bores et al. (2018a) plantea algunas características que debieran tener las CoP para producir beneficios en las prácticas profesionales, tales como la necesidad de que existan normas que promuevan la colaboración y la responsabilidad colectiva, la articulación de actividades que promuevan el acceso a la práctica y la confianza y familiaridad interpersonal.

Se ha indicado en las diversas investigaciones que la participación en CoP tanto para docentes como estudiantes, se presenta como una alternativa de formación y de aprendizaje colaborativo que permite a sus miembros generar y compartir conocimiento sobre un tema específico (Bedoya et al., 2018). De acuerdo con Arroyo et al. (2018) los entornos virtuales deben ser el principal espacio de comunicación entre la comunidad virtual que constituye la universidad o institución que se está formando. Estos espacios deben permitir la relación de los estudiantes entre ellos; de los estudiantes con los 
profesores y de los profesores entre sí, además de cualquier otro integrante de la comunidad universitaria.

Aldana y Martínez, (2018) y Bores et al. (2018b) detallan una serie de motivaciones para los profesores respecto a las comunidades de práctica online, y aseguran que estos se involucran, entre otras razones, para mejorar sus habilidades de enseñanza y su conocimiento pedagógico.

Smith et al. (2017) consideran que, al introducir la tecnología basadas en la web en una $\mathrm{CoP}$, suelen servir como un medio para garantizar la participación de los alumnos entre sí con el fín de generar conocimientos y recursos comunitarios que formen su práctica social. Sin embargo, el simple hecho de agregar estos espacios interactivos a un entorno de aprendizaje en línea no garantiza que las interacciones resultantes respalden los tipos de significados necesarios para el desarrollo de una CoP.

Las CoP pueden guiar el desarrollo de intervenciones para hacer el proceso educativo más eficaz y pueden ayudar tanto a los estudiantes como a los docentes a afrontar mejor la complejidad de la educación universitaria (Cruess et al., 2018). Entre las diversas ventajas de la $\mathrm{CoP}$, se tiene que permiten al participante regular sus intervenciones sabiendo que la comunicación se establece de forma asincrónica, siendo posible participar en momentos de mayor disponibilidad, ya sea simplemente leyendo las aportaciones de otros docentes o añadiendo comentarios a los hilos de debate, sin que sea necesaria la intervención del docente o administrador de la comunidad. Sobre todo, porque el conocimiento que se genera, en su práctica online permanece en el tiempo al quedar grabado en la plataforma, estando a disposición de los participantes de la comunidad, en cualquier momento para su consulta o para el proseguimiento de una discusión anterior (Liberatore et al., 2018).

Ahora bien, entre las limitaciones, se encuentra el grado de dificultad que puede presentarse al docente, lograr una participación acorde en las conversaciones, profundizando lo suficiente como para descubrir y comprometerse honestamente con algunos de los prejuicios inconscientes hacia la forma como los participantes piensan e interactúan entre sí. Debe tenerse presente la noción de que el profesorado es un aprendiz (Costino, 2018). 
El docente debe tener una adecuada preparación para poder enfrentar los diferentes niveles y tipos de experiencias o conocimientos previos que tienen los participantes, para crear un adecuado espacio de equidad. Sobre todo, cuando en algunos se tiene estructuras desiguales que producen negación y desestimación, asimismo cuando se emplea "lenguaje inapropiado" para describir a los estudiantes, que puede poner a algunos miembros del profesorado a la defensiva. Una forma de evitar este tipo de inconvenientes, es publicar las directrices de conducta dentro del grupo, responder rápidamente a los comentarios inapropiados, pro parte del docente cuando es el moderador o administrador del grupo (Costino, 2018; Liberatore et al., 2018).

El profesorado debe estar en constante aprendizaje, y si se desarrolla profesionalmente utilizando este método, observa en él un componente de libertad que le permite seleccionar aquellas temáticas más afines, al mismo tiempo que puede regular su participación y variar la intensidad y frecuencia de la misma de acuerdo a sus necesidades e intereses, aunque esta flexibilidad y falta de objetivos claros o tareas a cumplir, pueda ocasionar el abandono o la falta de participación activa.

Asimismo, se ha visto que los individuos que participan de la comunidad, pasan de la periferia legítima a la participación plena, adquiriendo la identidad de los miembros de la comunidad y aceptando las normas de la comunidad (Costino, 2018; Cruess et al., 2018; Liberatore et al., 2018; Groves y O'Shea, 2019).

\section{Conclusiones}

- Las comunidades de práctica virtuales se han convertido en una herramienta útil para el intercambio de conocimiento y experiencias en los últimos años, aportan ventajas que se añaden a las actividades colaborativas tradicionales.

- La construcción colectiva de conocimiento que se logra en una comunidad de práctica tiene un impacto positivo en las prácticas profesionales de los docentes, así como también tiene efectos positivos en el desarrollo académico de los alumnos. 


\section{Referencias}

Aldana, U., \& Martinez, D. (2018). The Development of a Community of Practice for Educators Working with Newcomer. Theory Into Practice, 57(2). https://doi.org/10.1080/00405841.2018.1425813

Arroyo, Z., Fernández, S., Barreto, L., \& Paz, L. (2018). Entornos virtuales de aprendizaje en comunidades de práctica de docentes universitarios del Ecuador. Revista Ensayos Pedagógicos, 13(2), 185. https://doi.org/10.15359/rep.13-2.9

Barab, S. A., Barnett, M., \& Squire, K. (2002). Developing an empirical account of a community of practice: Characterizing the essential tensions. Journal of the Learning Sciences, 11(4), 489-542. https://doi.org/10.1207/S15327809JLS1104_3

Bedoya-González, J., Betancourt-Cardona, M. O., \& Villa-Montoya, F. (2018). Creación de una comunidad de práctica para la formación de docentes en la integración de las tic a los procesos de aprendizaje y enseñanza de lenguas extranjeras. Íkala, Revista de Lenguaje y Cultura, 23(1), 121-139. https://doi.org/10.17533/udea.ikala.v23n01a09

Bores, D., González, G., \& García, A. (2018a). (Re)Produce: desarrollo profesional docente en una comunidad de práctica virtual informal de Educación Física. Sportis. Scientific Journal of School Sport, Physical Education and Psychomotricity, 4(3), 480-507. https://doi.org/10.17979/sportis.2018.4.3.3301

Bores, D., González, G., \& García, A. (2018b). Barreras y motivaciones para la participación en una comunidad de práctica virtual de docentes de Educación Física : el caso de (Re) Produce. Ágora para la educación física y el deporte, 20(2), 368-391. https://doi.org/10.24197/aefd.2-3.2018.368-391

Bosman, L., \& Voglewede, P. (2019). How Can a Faculty Community of Practice Change Classroom Practices? College Teaching, 67(3), 177-187. https://doi.org/10.1080/87567555.2019.1594149 
Costino, K. (2018). Equity-Minded Faculty Development: An Intersectional IdentityConscious Community of Practice Model for Faculty Learning. Metropolitan Universities, 29(1). https://doi.org/10.18060/22170

Cruess, R. L., Cruess, S. R., \& Steinert, Y. (2018). Medicine as a Community of Practice: Implications for Medical Education. Academic Medicine, 93(2), 185191. https://doi.org/10.1097/ACM.0000000000001826

de Carvalho-Filho, M. A., Tio, R. A., \& Steinert, Y. (2020). Twelve tips for implementing a community of practice for faculty development. Medical Teacher, 42(2), 143-149. https://doi.org/10.1080/0142159X.2018.1552782

García, J., Castillo, A., \& Aguilera, J. R. (2008). Sociedad del conocimiento y políticas neoliberales: la escuela bajo acoso. Revista Latinoamericana de Estudios Educativos, 38, 35-59. http://www.redalyc.org/pdf/270/27012437003.pdf

Groves, O., \& O'Shea, S. (2019). Learning to 'be' a university student: First in family students negotiating membership of the university community. International Journal of Educational Research, 98, 48-54.

https://doi.org/10.1016/j.ijer.2019.08.014

Hafeez, K., Alghatas, F. M., Foroudi, P., Nguyen, B., \& Gupta, S. (2019). Knowledge sharing by entrepreneurs in a virtual community of practice (VCoP). Information Technology and People, 32(2), 405-429. https://doi.org/10.1108/ITP-09-2016$\underline{0202}$

Hara, N. (2009). Communities of Practice (Vol. 13). Springer Berlin Heidelberg. https://doi.org/10.1007/978-3-540-85424-1

Kensington-Miller, B. (2018). Surviving the first year: new academics flourishing in a multidisciplinary community of practice with peer mentoring. Professional Development in Education, 44(5), 678-689. https://doi.org/10.1080/19415257.2017.1387867

Li, L.C., Grimshaw, J.M., Nielsen, C., Judd, M., Coite, P.C., \& Graham, I. D. (2009) Evolution of Wenger's concept of community of practice. Implementation Science 4(11). https://doi.org/10.1186/1748-5908-4-11 
Liberatore, A., Bowkett, E., MacLeod, C. J., Spurr, E., \& Longnecker, N. (2018). Social

Media as a Platform for a Citizen Science Community of Practice. Citizen

Science: Theory and Practice, 3(1), 3. https://doi.org/10.5334/CSTP.108

Paz, L., Hernández, E., \& Van de Water, H. (2016). Los retos de la educación superior en el siglo XXI. Revista Conrado, 15(55), 17-24.

https://conrado.ucf.edu.cu/index.php/conrado/article/view/343

Scarso, E., Bolisani, E., \& Salvador, L. (2009). A systematic framework for analysing the critical success factors of communities of practice. Journal of Knowledge Management, 13(6), 431-447. https://doi.org/10.1108/13673270910997105

Smith, S. U., Hayes, S., \& Shea, P. (2017). A critical review of the use of Wenger's community of practice $(\mathrm{CoP})$ theoretical framework in online and blended learning research, 2000-2014. Online Learning Journal, 21(1), 209-237. https://doi.org/10.24059/olj.v21i1.963

Vegessi, M., \& Shaw, J. M. (2018). Teaching Engineering Innovation, Design, and Leadership in a Community of Practice. Proceedings of the Canadian Engineering Education Association (CEEA), 1-7. https://doi.org/10.24908/pceea.v0i0.13058

Wenger, E. (2000). Communities of practice and social learning systems : the career of a concept A social systems view on learning : communities of practice as social learning systems. Organization2, 7(2), 225-264. https://link.springer.com/chapter/10.1007/978-1-84996-133-2_11

Wing Lai, K., Pratt, K., Anderson, M., \& Stigter, J. (2006). Literature Review and Synthesis : Online Communities of Practice. New Zeland: Research Division Wahanga Mahi Rangahau Misnistry of Education. https://www.researchgate.net/publication/242678191_Literature_Review_and_Sy nthesis_Online_Communities_of_Practice 